УДК 541.123 .3

DOI: https://doi.org/10.17308/kcmf.2019.21/763

Поступила в редакцию 19.04.2019

Подписана в печать 15.05.2019

\title{
АНАЛИЗ ФАЗОВЫХ РАВНОВЕСИЙ В ТРОЙНОЙ СИСТЕМЕ Ge-P-Sn
}

\author{
(c) 2019 Г. В. Семенова $\bowtie$, Т. А. Леонтьева, Т. П. Сушкова \\ Воронежский государственный университет \\ Университетская пл., 1, 394018 Воронеж, Российская Федерация
}

\begin{abstract}
Аннотация. На основании анализа характера фазовых равновесий в двойных системах, ограняющих диаграмму состояний тройной системы Ge - P - Sn, предложены теоретически возможные схемы ее фазового субсолидусного разграничения. Исследование методом рентгенофазового анализа образцов, принадлежащих политермическим сечениям $\mathrm{Sn}_{4} \mathrm{P}_{3}$ $\mathrm{Ge}, \mathrm{Sn}_{4} \mathrm{P}_{3}-\mathrm{GeP}$, показало, что разделение трехкомпонентной диаграммы состояния ниже солидуса осуществляется с помощью сечений $\mathrm{Sn}_{4} \mathrm{P}_{3}$-Ge, $\mathrm{Sn}_{4} \mathrm{P}_{3}-\mathrm{GeP}$ и $\mathrm{SnP}_{3}$-GeP. Построенная по данным дифференциального термического анализа фазовая диаграмма сечения $\mathrm{Sn}_{4} \mathrm{P}_{3}$ Ge представляет диаграмму эвтектического типа с координатами эвтектической точки $800 \mathrm{~K}, 15 \mathrm{~mol} \% \mathrm{Ge}$.
\end{abstract}

Ключевые слова: фазовые равновесия, система Ge-P-Sn, фазовое субсолидусное разграничение.

\section{ВВЕДЕНИЕ}

Для применения в современных электронных устройствах все более привлекательными становятся 2D материалы за счет новых электронных свойств, которые могут возникать из-за уменьшенной размерности и квантового ограничения носителей заряда. Много работ направлено на поиск материалов, характеризующихся слоистой структурой, позволяющих получать химически стабильные атомные слои без поверхностных оборванных связей $[1,2]$. Помимо графена, кремний и германий также могут быть получены в виде атомно тонких слоев (силикен [3], германен [4] соответственно) либо химическим осаждением на подложке [5], либо механическим расслоением объемных трехмерных кристаллов [6]. Бинарные соединения элементов группы IV (Si, Ge, Sn) и группы V (P, As) образуют слоистые структуры, в которых двумерные слои с ковалентной связью связаны слабыми силами Ван-дерВаальса [7], и с этой точки зрения могут считаться перспективными 2D материалами.

Следует, однако, отметить, что получение кристаллов соединений этого класса сопряжено со значительными сложностями. В частности, фосфид германия разлагается по перитектической схеме при $998 \mathrm{~K}$, при этом развивается

$\triangle$ Семенова Галина Владимировна e-mail: semen157@chem.vsu.ru давление $26.3 \cdot 10^{3} \mathrm{hPa}$ [8]. В [9] была предпринята попытка получить образцы $\mathrm{GeP}$ из раствороврасплавов в олове, что значительно смягчало условия синтеза. Тем не менее, полученные сплавы в качестве сопутствующей фазы содержали фосфиды олова. Исследование фазовых равновесий и построение диаграммы состояний тройной системы $\mathrm{Ge}-\mathrm{P}-\mathrm{Sn}$ позволило бы с качественно иных позиций подойти к получению как объемных, так и двумерных образцов фосфида германия, а также определить возможность легирования их оловом.

Цель настоящей работы заключалась в анализе характера фазовых равновесий в тройной системе $\mathrm{Ge}-\mathrm{P}-\mathrm{Sn}$, исследовании политермического сечения $\mathrm{Ge}-\mathrm{Sn}_{4} \mathrm{P}_{3}$ методами рентгенофазового и дифференциального термического анализа.

\section{ЭКСПЕРИМЕНТАЛЬНАЯ ЧАСТЬ}

Синтез сплавов осуществляли однотемпературным методом, используя фосфор ОСЧ-9-5, олово марки ОВЧ-000 и поликристаллический зонноочищенный германий ГОСТ 16154-80. Синтез образцов, принадлежащих сечению $\mathrm{Sn}_{4} \mathrm{P}_{3}-$ $\mathrm{Ge}$, проводили в два этапа. На первом этапе был получен фосфид олова $\mathrm{Sn}_{4} \mathrm{P}_{3}$ прямым сплавлением исходных веществ. Режим нагрева печи был следующим: нагревание до 673 K со скоростью $7.5 \mathrm{~K} / \mathrm{min}$, далее выдержка $60 \mathrm{~min}$, затем нагре- 
вание до $893 \mathrm{~K}$ со скоростью $1.2 \mathrm{~K} / \mathrm{min}$, выдержка 30 минут. На втором этапе был осуществлен синтез сплавов тройной системы, составы которых соответствуют политермическому сечению $\mathrm{Ge}-\mathrm{Sn}_{4} \mathrm{P}_{3}$. Печь нагревали до температуры 1238 К со скоростью $5.2 \mathrm{~K} / \mathrm{min}$. Из-за сложности получения фосфида германия синтез образцов сечения $\mathrm{Sn}_{4} \mathrm{P}_{3}$-GeP проводили в один этап из простых веществ. При этом при температуре 673 К осуществляли изотермическую выдержку в течение 3 часов для максимально возможного взаимодействия расплава олова с фосфором, после чего температуру увеличивали до $1233 \mathrm{~K}$ со скоростью $1.3 \mathrm{~K} / \mathrm{min}$. Для гомогенизации образцов и приведения системы в состояние, близкое к равновесному, все образцы отжигали при температуре $753 \mathrm{~K}$ в течение 120 часов.

Дифференциальный термический анализ (ДТА) образцов осуществляли на установке с программируемым нагревом печи при скорости нагревания $5 \mathrm{~K} / \mathrm{min}$, используя ПИД-регуляторы ОВЕН ТРМ-151 и ТРМ-202. Сигнал, полученный с предварительно отградуированных хромельалюмелевых термопар, оцифровывался и обрабатывался при помощи компьютерной программы «MasterSCADA». Погрешность определения температуры фазовых переходов методом ДТА не превышала $\pm 2 \mathrm{~K}$.

Рентгенофазовый анализ (РФА) проводили на дифрактометре ARL X'TRA в геометрии $\Theta-\Theta$ с фокусировкой по Бреггу-Брентано; использовалась рентгеновская трубка с медным анодом, $\lambda\left(\mathrm{Cu}-K_{\alpha 1}\right)=0.1541 \mathrm{~nm} ; \lambda\left(\mathrm{Cu}-K_{\alpha 2}\right)=0.1544 \mathrm{~nm} ;$ шаг

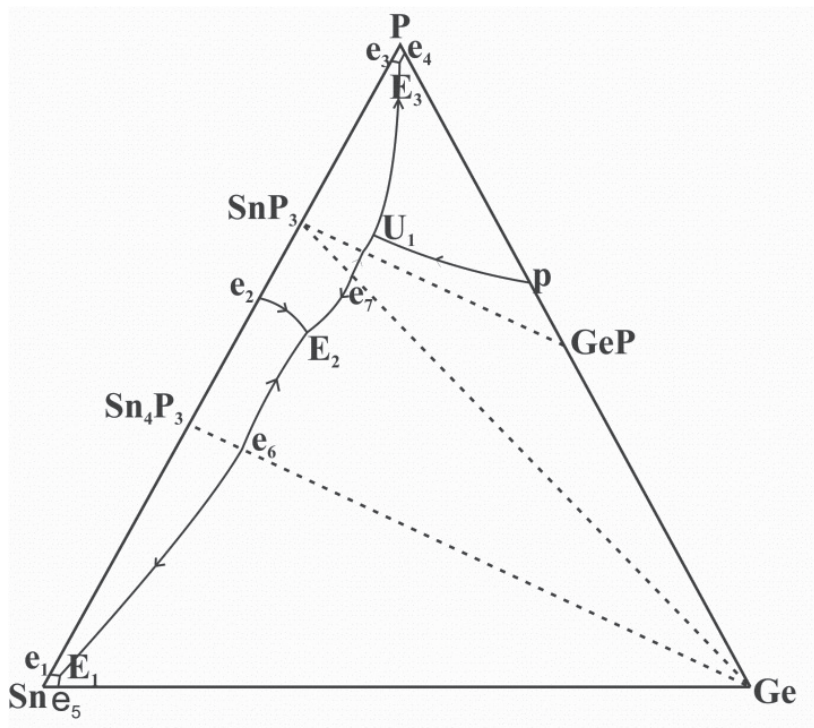

$a$ съемки $0.04^{\circ}$, время выдержки - 3 секунды. Погрешность определения межплоскостных расстояний $d_{\mathrm{hkl}}$ не превышала $5 \cdot 10^{-4} \mathrm{~nm}$.

\section{РЕЗУЛЬТАТЫ И ИХ ОБСУЖДЕНИЕ}

Характер фазовых равновесий в тройной системе $\mathrm{Ge}-\mathrm{P}-\mathrm{Sn}$ достаточно сложен, поскольку помимо фосфида германия, образующегося по перитектической схеме, в бинарной системе $\mathrm{Sn}$ - P также присутствует несколько промежуточных фаз. Согласно $[10,11]$ в системе существуют две фазы $\mathrm{Sn}_{4} \mathrm{P}_{3}$ и $\mathrm{SnP}_{3}$; еще одна промежуточная фаза $\mathrm{Sn}_{3} \mathrm{P}_{4}$ распадается по перитектоидной схеме. Фазовая диаграмма системы $\mathrm{Ge}-\mathrm{Sn}$ характеризуется наличием вырожденной эвтектики [12]. Для упрощения последующего анализа фазовых равновесий в тройной системе следует провести субсолидусное фазовое разграничение диаграммы состояний, тем более при наличии в бинарных системах нестехиометрических фаз и твердых растворов на основе компонентов.

На рис. $1 a, b$ представлены возможные схемы равновесий в этой системе. Наличие в двойных системах $\mathrm{Sn}-\mathrm{Ge}$ и $\mathrm{Sn}-\mathrm{P}$ эвтектических точек $\left(\mathrm{e}_{5}, \mathrm{e}_{1}\right)$, близких по составу и температуре к чистому олову, позволяет предположить наличие в тройной системе эвтектической точки $\mathrm{E}_{1}$. В ней осуществляется процесс $\mathrm{L} \leftrightarrow \mathrm{Sn}+\mathrm{Ge}+\mathrm{Sn}_{4} \mathrm{P}_{3}$. Эвтектика в системе $\mathrm{Ge}-\mathrm{P}$ также является вырожденной, а в системе олово - фосфор эвтектическая точка реализуется, вероятно, при высоких концентрациях фосфора. Соответственно этому, в области высокого содержания фосфора также

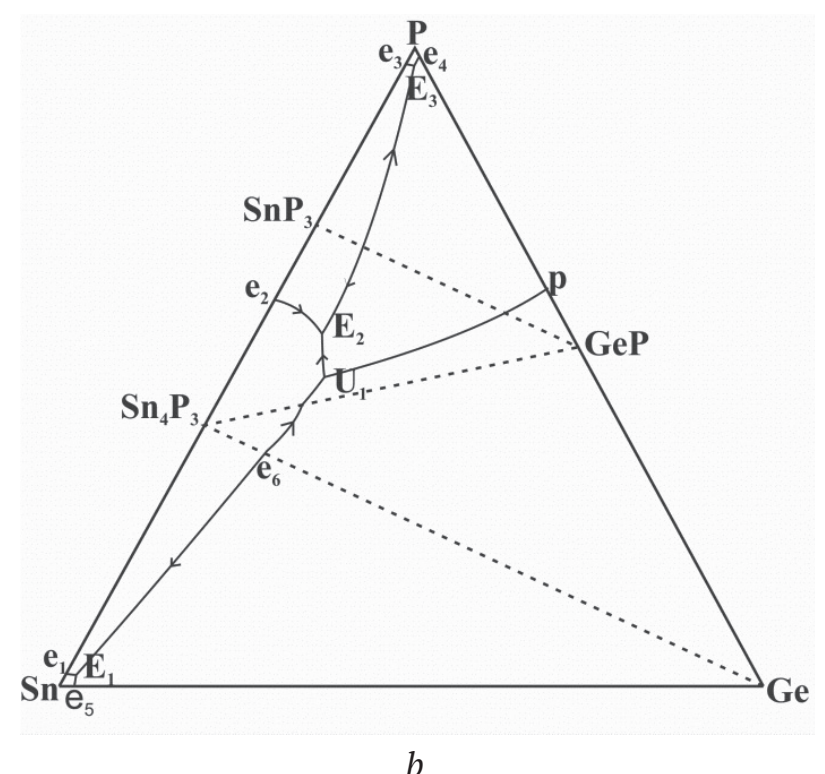

$b$

Рис. 1. Схемы фазовых равновесий в системе $\mathrm{Sn}-\mathrm{Ge}-\mathrm{P}: a-$ вариант $1 ; b-$ вариант 2

[Fig. 1. The schemes of the phase equilibria in the $\mathrm{Sn}-\mathrm{Ge}-\mathrm{P}$ system: $a$ - option $1 ; b$-option 2] 
осуществляется равновесие эвтектического характера $\mathrm{L} \leftrightarrow \mathrm{P}+\mathrm{GeP}+\mathrm{SnP}_{3}\left(\right.$ т. $\left.\mathrm{E}_{3}\right)$.

Поскольку фосфид германия разлагается по перитектической схеме, очевидно, что вдоль линии трехфазного равновесия $\mathrm{pU}$ будет осуществляться процесс взаимодействия расплава и германия с образованием GeP. В случае реализации схемы 1 этот процесс будет заканчиваться четырехфазным равновесием $\mathrm{L}+\mathrm{Ge} \leftrightarrow \mathrm{GeP}+\mathrm{SnP}_{3}$ $\left(\right.$ т. $\left.U_{1}\right)$. Соответственно этому выше по температуре этому процессу будет предшествовать совместная кристаллизация $\mathrm{SnP}_{3}$ и германия, а ниже по температуре осуществляться процесс $\mathrm{L} \leftrightarrow \mathrm{GeP}+\mathrm{SnP}_{3}\left(\mathrm{U}_{1} \mathrm{E}_{3}\right)$. В точке $\mathrm{E}_{2}$ четырехфазный процесс $\mathrm{L} \leftrightarrow \mathrm{SnP}_{3}+\mathrm{Ge}+\mathrm{Sn}_{4} \mathrm{P}_{3}$ носит эвтектический характер. Таким образом, этот вариант предполагает разделение фазовой диаграммы при субсолидусных температурах с помощью разрезов $\mathrm{Ge}-\mathrm{SnP}_{3}$ и $\mathrm{Ge}-\mathrm{Sn}_{4} \mathrm{P}_{3}$.

Для схемы, представленной на рис. $1 b$, трехфазный процесс L + Ge $\leftrightarrow$ GeP заканчивается в т. $\mathrm{U}_{1}$, где при взаимодействии германия с расплавом образуются две твердые фазы - фосфид германия и фосфид олова состава $\mathrm{Sn}_{4} \mathrm{P}_{3}$. Соответственно этому, ему предшествует совместное выделение германия и $\mathrm{Sn}_{4} \mathrm{P}_{3}$ (линия е $\mathrm{U}_{1}$ ), а ниже по температуре следует эвтектическое равновесие L $\leftrightarrow \mathrm{Sn}_{4} \mathrm{P}_{3}+\mathrm{GeP}$ (линия е $\mathrm{E}_{2}$ ). Эвтектическое четырехфазное равновесие совместной кристаллизации фосфидов олова $\mathrm{Sn}_{4} \mathrm{P}_{3}$ и $\mathrm{SnP}_{3}$, а также фосфида германия имеет место в т. $\mathrm{E}_{2}$.

В обеих схемах фазовое субсолидусное разделение осуществляет разрез $\mathrm{Ge}-\mathrm{Sn}_{4} \mathrm{P}_{3}$. Для подтверждения этого было осуществлено экспериментальное исследование сплавов, состав которых отвечает этому сечению.

На рис. 2 представлены дифрактограммы сплавов, составы которых отвечают исследуемому разрезу. На дифрактограммах фиксируются рефлексы только двух фаз - германия и фосфида олова, интенсивность которых планомерно увеличивается по мере роста содержания соответствующей фазы в сплаве. Расчет параметра кристаллической решетки германия по полученным данным показал, что он практически не меняется в зависимости от состава сплава и составляет $5.66272 \AA$ А. Это свидетельствует о том, что тройной твердый раствор на основе германия практически реализуется в очень узком концентрационном интервале. Это согласуется с тем, что твердофазная растворимость олова в германии составляет менее, чем $1 \mathrm{~mol} \%$, а максимальная растворимость фосфора в германии не превышает $0.45 \mathrm{~mol} \%$ при 873 K [10]. В целом, результа- ты изучения сплавов сечения $\mathrm{Ge}-\mathrm{Sn}_{4} \mathrm{P}_{3}$ методом рентгенофазового анализа позволяют говорить о том, что разрез может осуществлять фазовое разделение диаграммы тройной системы.

Это подтверждается и данными термографических исследований сплавов. На термограммах образцов фиксируется два эндотермических эффекта, причем низкотемпературный эффект реализуется при одной и той же температуре 800 К. Второй эффект, отвечающий ликвидусу на фазовой диаграмме, особенно четко виден на кривых охлаждения. Термограммы некоторых сплавов представлены на рис. 3.

Фазовая диаграмма сечения $\mathrm{Ge}-\mathrm{Sn}_{4} \mathrm{P}_{3}$ представлена на рис. 4. При построении фазовой диаграммы разреза $\mathrm{Ge}-\mathrm{Sn}_{4} \mathrm{P}_{3}$ некоторые линии нанесены с учетом теоретического анализа тройной системы, что связано с трудностями фиксирования методом ДТА эффектов, близких по температуре. Учитывая тот факт, что в системе олово - фосфор фосфид $\mathrm{Sn}_{4} \mathrm{P}_{3}$ участвует в синтектическом равновесии, характерное для бинарной системы расслоение жидкой фазы может оказать влияние на характер поверхности ликвидуса тройной диаграммы в целом, и, в частности, на вид рассматриваемого сечения. Логично предположить, что в тройной системе в области, прилегающей к стороне Sn-P, также может происходить разложение твердой фазы на две жидкости разного состава. В тройной системе этому моновариантному процессу должен отвечать трехфазный объем $\mathrm{L}_{1}+\mathrm{L}_{2}+\mathrm{Sn}_{4} \mathrm{P}_{3}$.

Положение участков ликвидуса для разреза было оценено путем расчета по уравнению Шредера - Ле-Шателье [13], при этом использовали следующие параметры стабильности: для германия $T_{F}=1210 \mathrm{~K}=37038 \mathrm{~J} / \mathrm{mol}$ [14]; для фосфида олова $T_{F}=838 \mathrm{~K} ; \Delta H_{F}=18400 \mathrm{~J} / \mathrm{mol}$ [15]. Участки ликвидуса, соответствующие первичной кристаллизации фосфида олова и германия пересекаются при температуре 785 K и составе сплава с содержанием германия 12 mol \%. Учитывая, что была использована модель идеальных растворов, можно считать, что расчетные данные (кривая 1) на рис. 4 удовлетворительно согласуются с полученными экспериментальными данными.

Для того, чтобы выбрать из двух схем, представленных на рис. 1, методом рентгенофазового анализа были исследованы образцы, принадлежащие политермическому сечению $\mathrm{Sn}_{4} \mathrm{P}_{3}-\mathrm{GeP}$. Как следует из представленных на рис. 5 данных, сплавы разреза представляют смесь трех фаз: $\mathrm{Sn}_{4} \mathrm{P}_{3}$, GeP и Ge. Таким образом, схема, пред- 

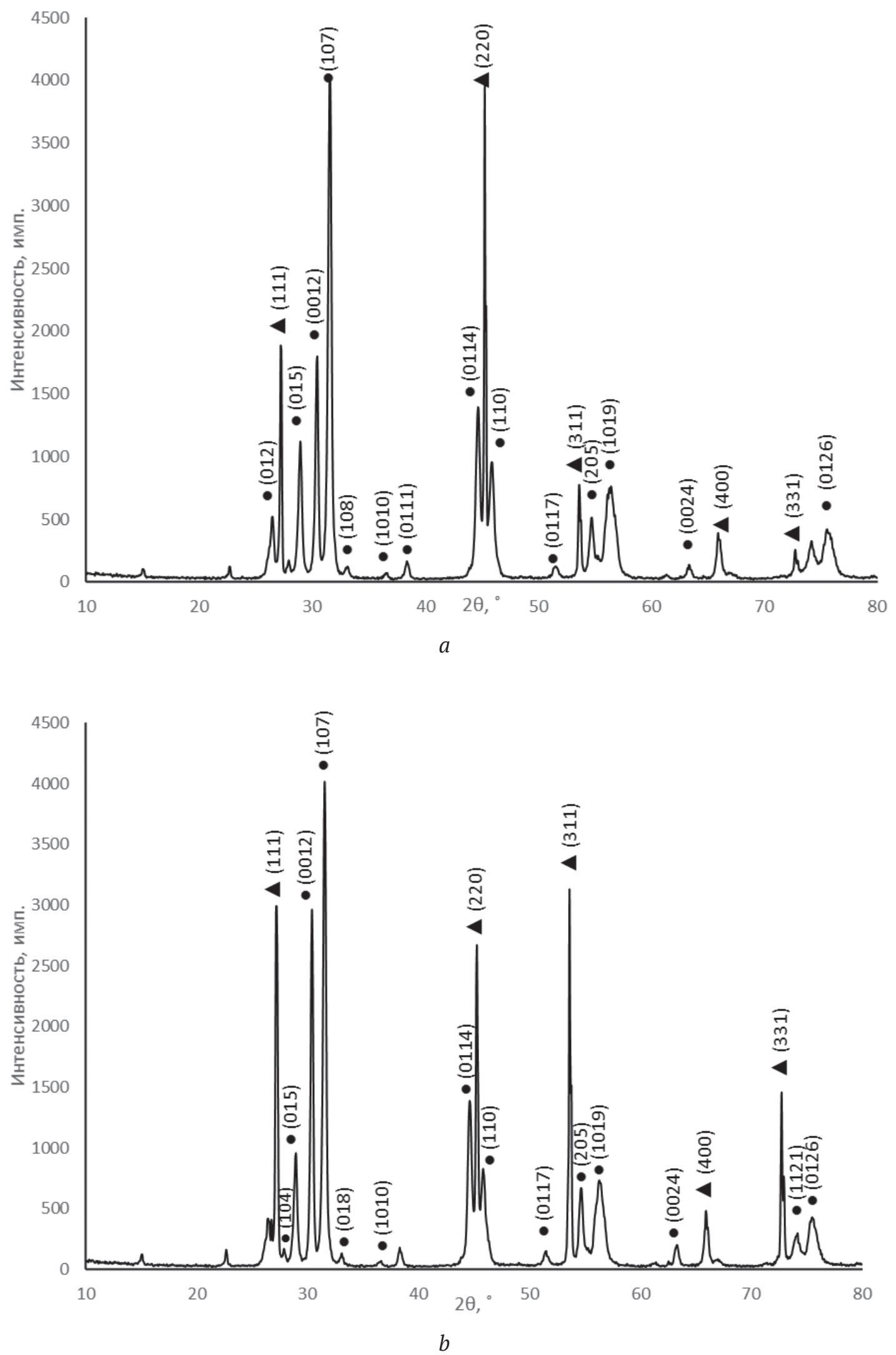

Рис. 2. Дифрактограммы сплавов политермического сечения $\mathrm{Ge}-\mathrm{Sn}_{4} \mathrm{P}_{3}: a-\left(\mathrm{Sn}_{4} \mathrm{P}_{3}\right)_{0.8} \mathrm{Ge}_{0.2}$; $b-\left(\mathrm{Sn}_{4} \mathrm{P}_{3}\right)_{0.6} \mathrm{Ge}_{0.4} ; c-\left(\mathrm{Sn}_{4} \mathrm{P}_{3}\right)_{0.4} \mathrm{Ge}_{0.6} ; d-\left(\mathrm{Sn}_{4} \mathrm{P}_{3}\right)_{0.2} \mathrm{Ge}_{0.8}$

[Fig. 2. X-ray powder diffraction patterns of alloys of polythermal section $\mathrm{Ge}-\mathrm{Sn}_{4} \mathrm{P}_{3}: a-\left(\mathrm{Sn}_{4} \mathrm{P}_{3}\right)_{0.8} \mathrm{Ge}_{0.2}$; $\left.b-\left(\mathrm{Sn}_{4} \mathrm{P}_{3}\right)_{0.6} \mathrm{Ge}_{0.4} ; c-\left(\mathrm{Sn}_{4} \mathrm{P}_{3}\right)_{0.4} \mathrm{Ge}_{0.6} ; d-\left(\mathrm{Sn}_{4} \mathrm{P}_{3}\right)_{0.2} \mathrm{Ge}_{0.8}\right]$ 

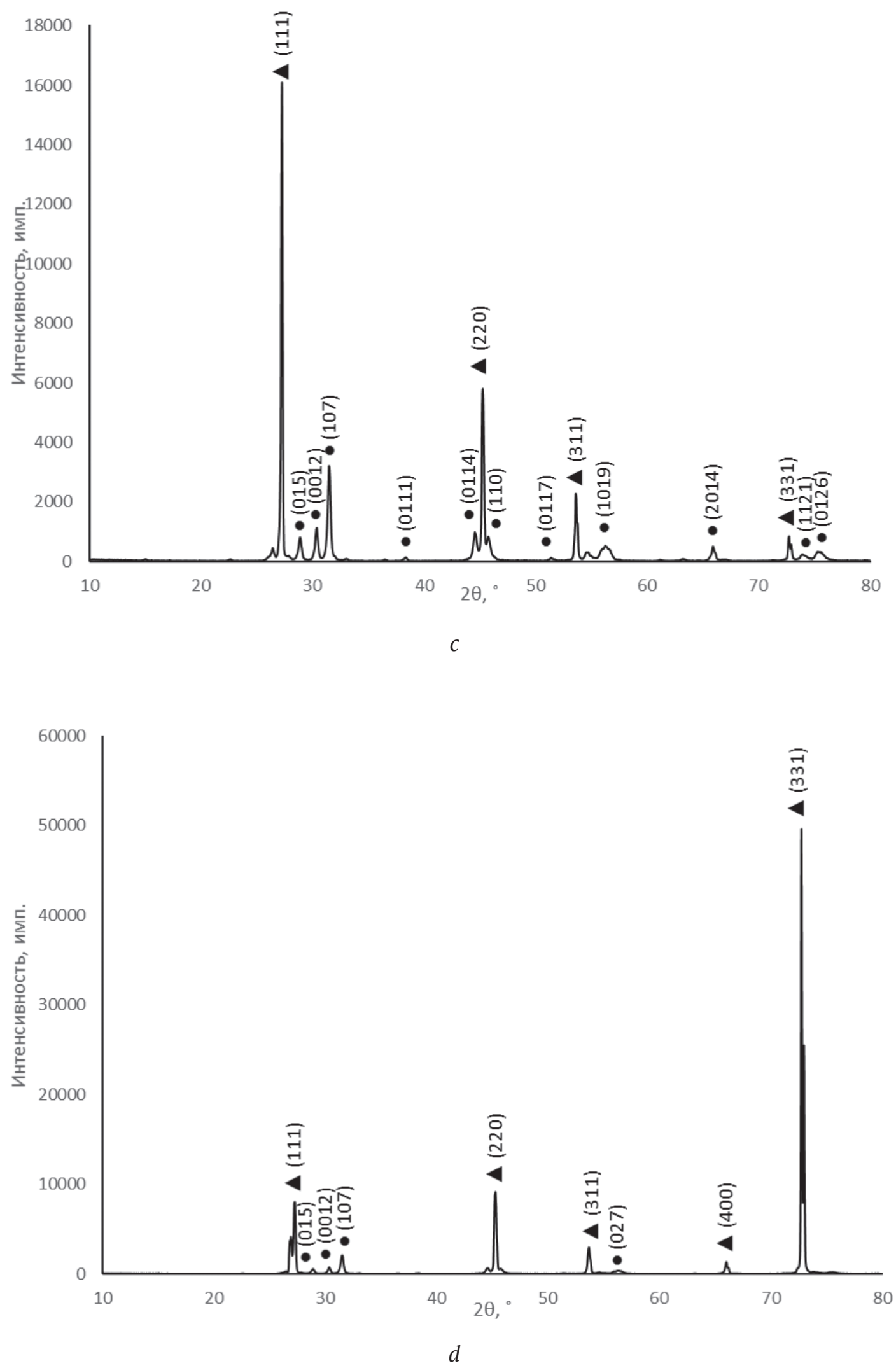


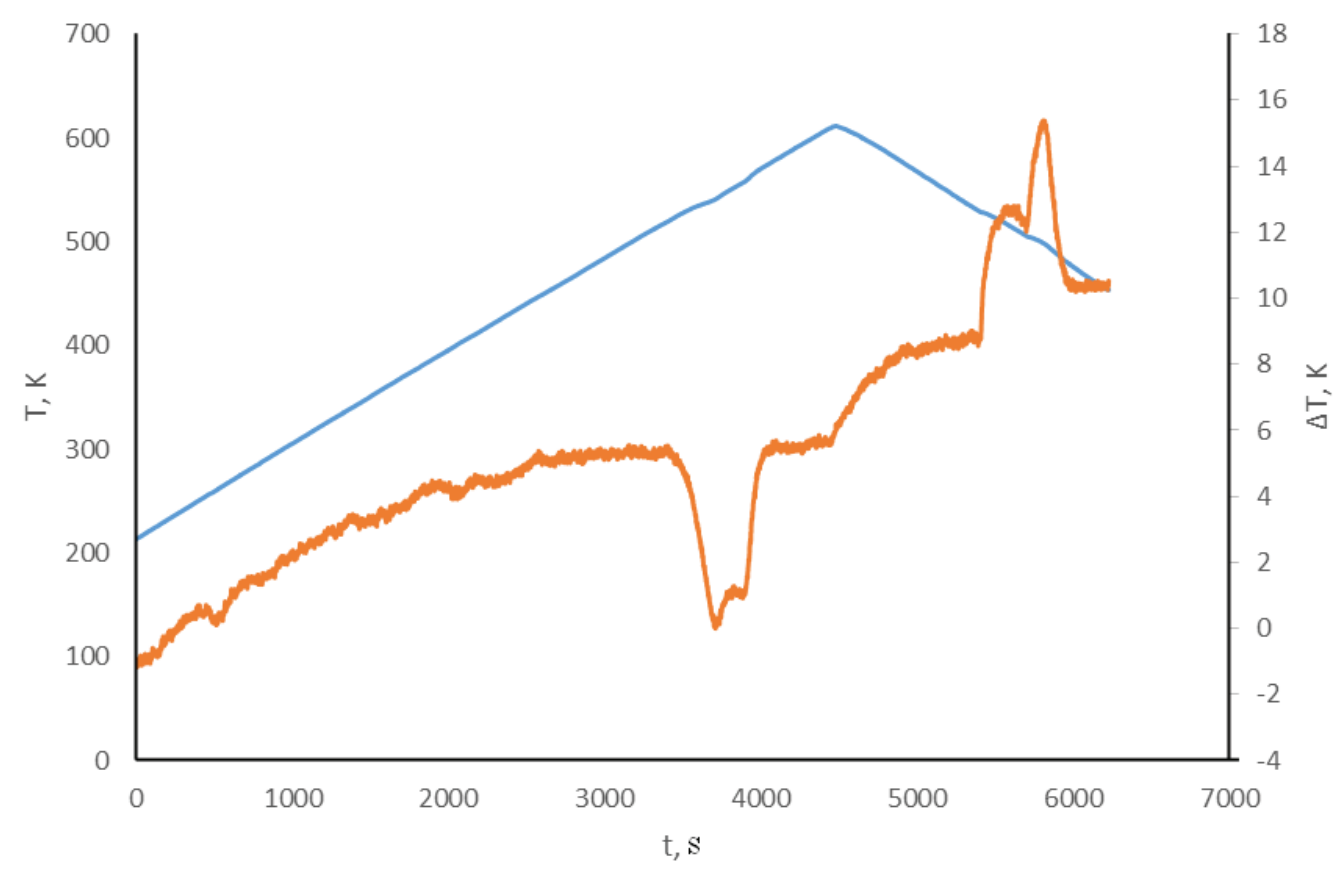

$a$

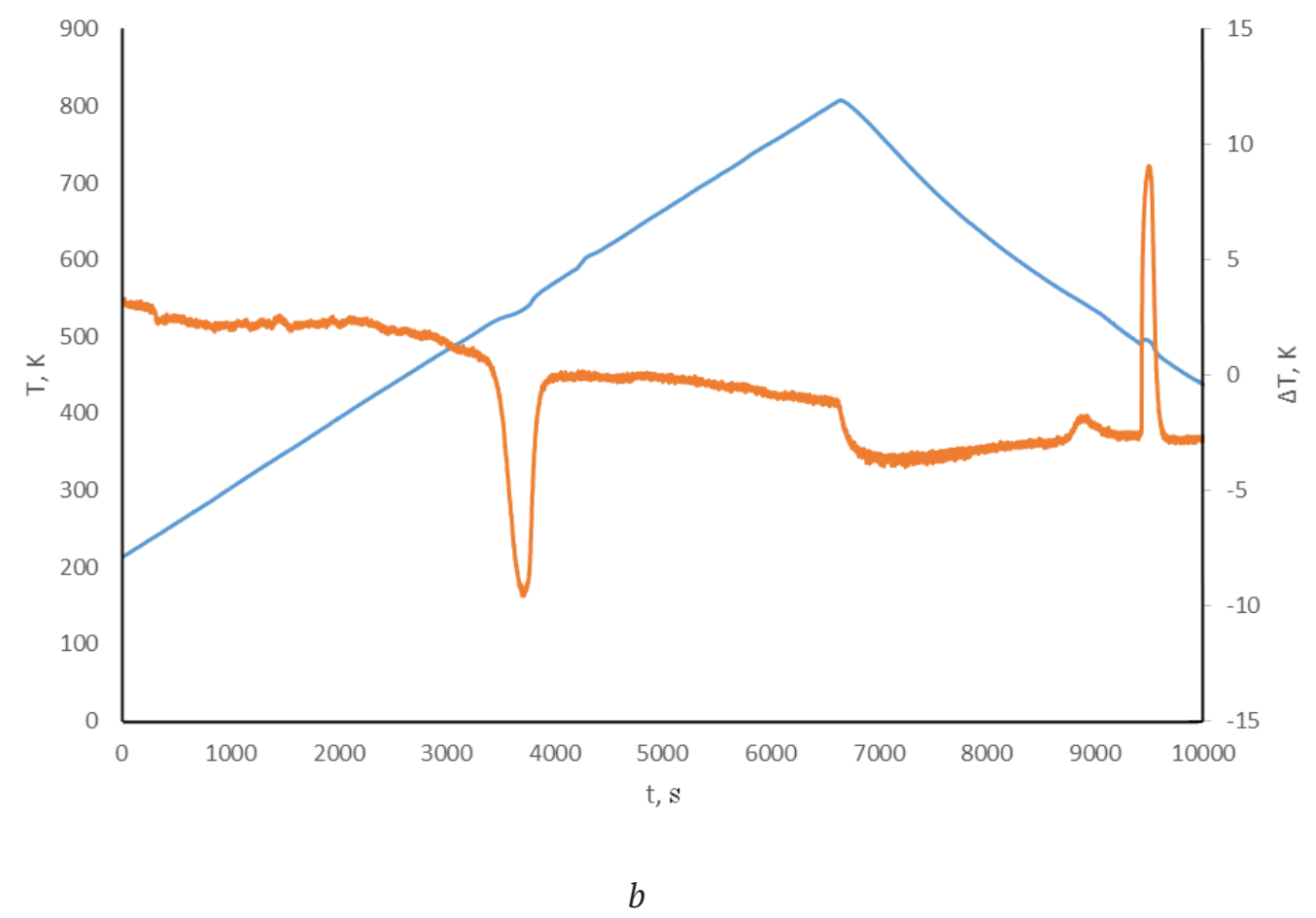

Рис. 3. Термограммы сплавов политермического сечения $\mathrm{Ge}-\mathrm{Sn}_{4} \mathrm{P}_{3}: a-\left(\mathrm{Sn}_{4} \mathrm{P}_{3}\right)_{0.9} \mathrm{Ge}_{0.1} ; b-\left(\mathrm{Sn}_{4} \mathrm{P}_{3}\right)_{0.8} \mathrm{Ge}_{0.2}$; $c-\left(\mathrm{Sn}_{4} \mathrm{P}_{3}\right)_{0.6} \mathrm{Ge}_{0.4} ; d-\left(\mathrm{Sn}_{4} \mathrm{P}_{3}\right)_{0.2} \mathrm{Ge}_{0.8}$

[Fig. 3. The curves of heating of alloys of polythermal section $\mathrm{Ge}-\mathrm{Sn}_{4} \mathrm{P}_{3}: a-\left(\mathrm{Sn}_{4} \mathrm{P}_{3}\right)_{0.9} \mathrm{Ge}_{0.1}$; $\left.b-\left(\mathrm{Sn}_{4} \mathrm{P}_{3}\right)_{0.8} \mathrm{Ge}_{0.2} ; c-\left(\mathrm{Sn}_{4} \mathrm{P}_{3}\right)_{0.6} \mathrm{Ge}_{0.4} ; d-\left(\mathrm{Sn}_{4} \mathrm{P}_{3}\right)_{0.2} \mathrm{Ge}_{0.8}\right]$ 


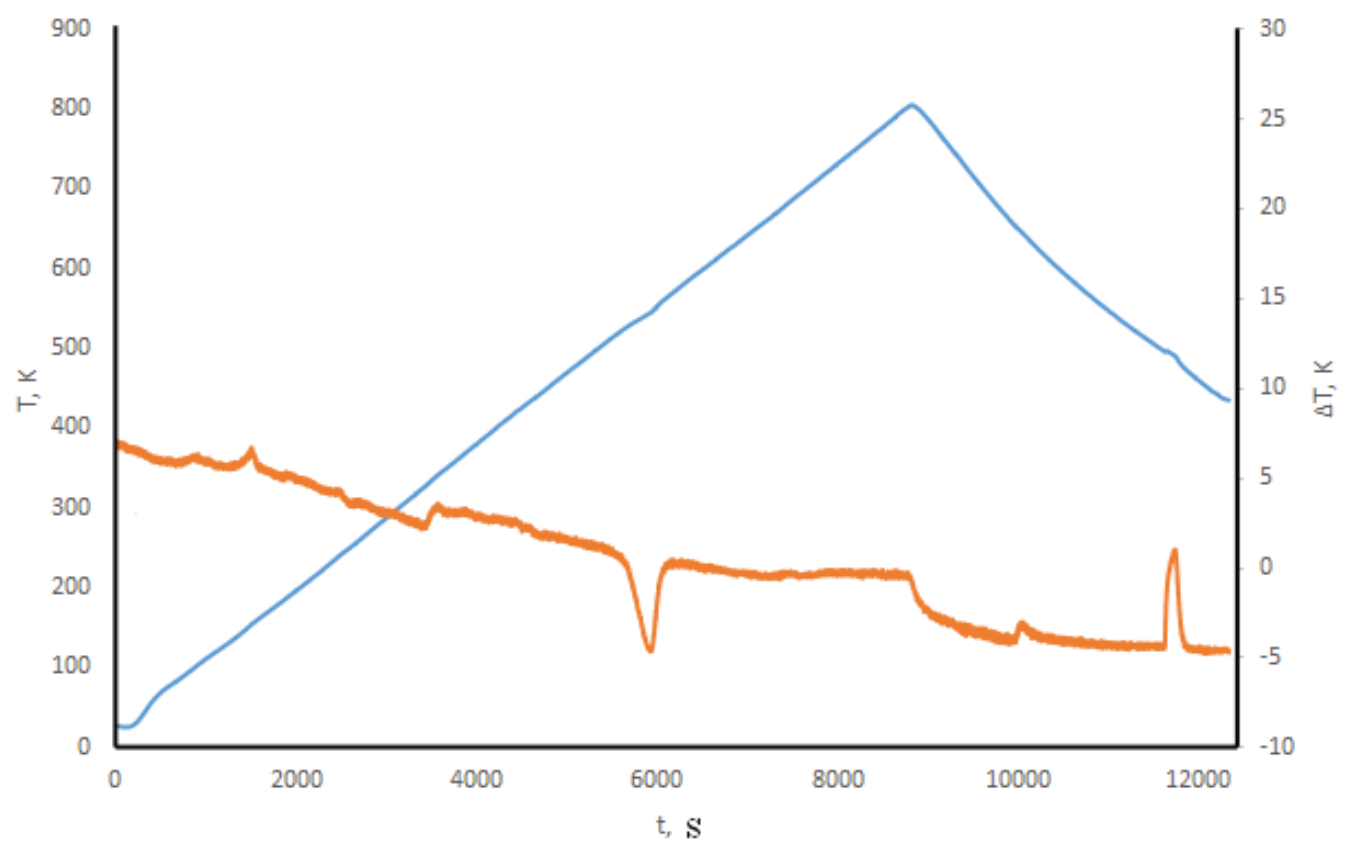

C

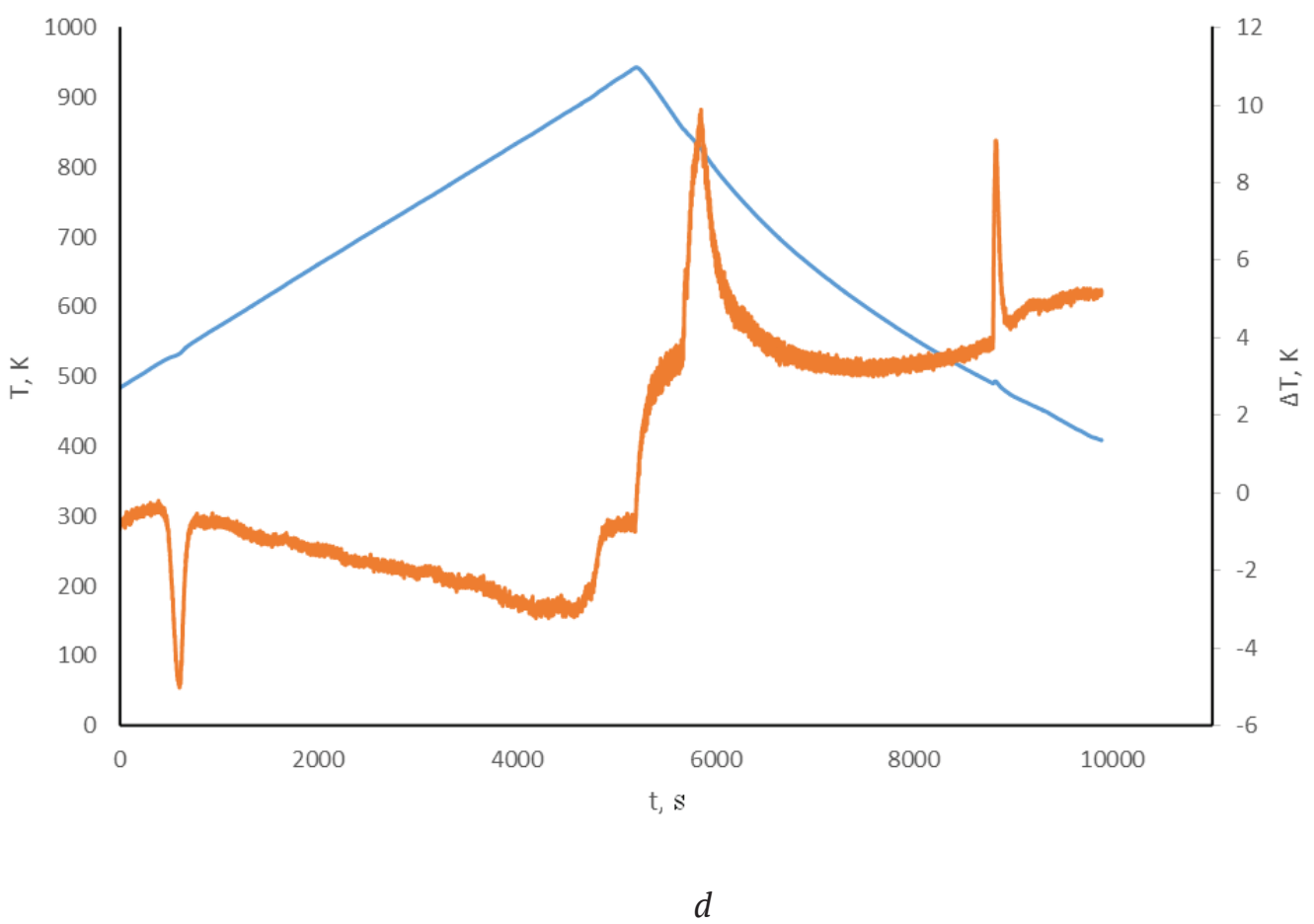




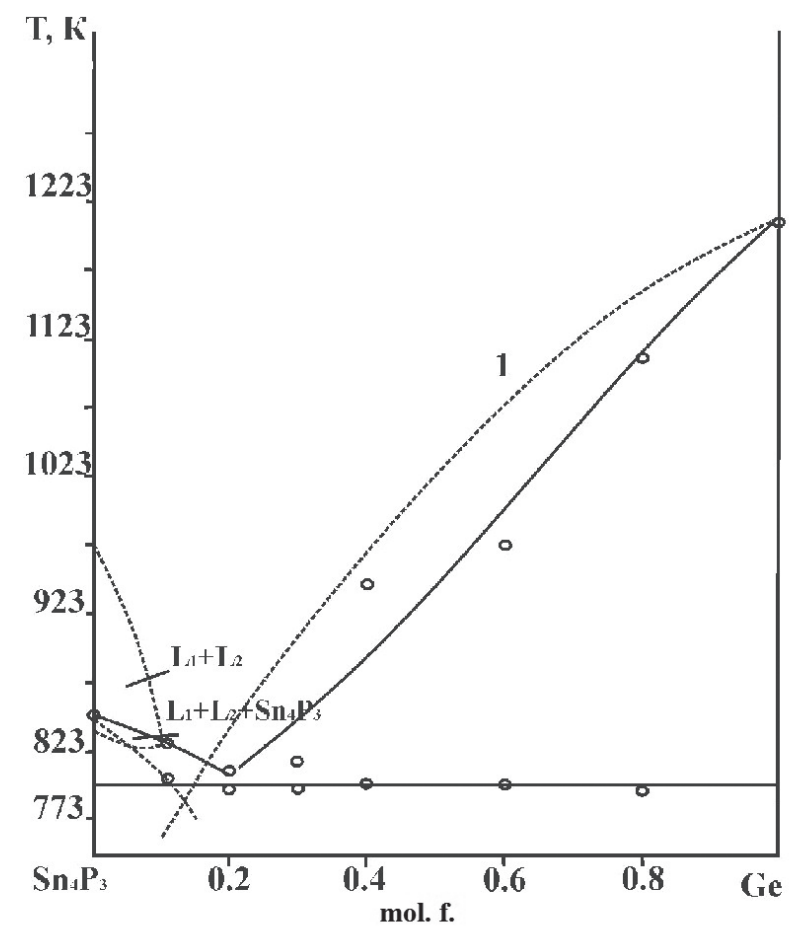

Рис. 4. Фазовая диаграмма политермического сечения $\mathrm{Ge}-\mathrm{Sn}_{4} \mathrm{P}_{3}$

[Fig. 4. Phase Diagram of the Ge-Sn ${ }_{4} P_{3}$ polythermic cross-section] ставленная на рис. $1 b$ является верной.

В табл. 1 систематизированы данные о четырех- и трехфазных процессах в тройной системе $\mathrm{Ge}-\mathrm{P}-\mathrm{Sn}$.

\section{ЗАКЛЮЧЕНИЕ}

Экспериментальное исследование методами дифференциального термического и рентгенофазового анализа сплавов политермического сечения $\mathrm{Sn}_{4} \mathrm{P}_{3}-\mathrm{GeP}$ тройной системы $\mathrm{Ge}-\mathrm{P}-\mathrm{Sn}$ показало, что образцы представляют собой эвтектическую смесь двух фаз - германия и фосфида олова $\mathrm{Sn}_{4} \mathrm{P}_{3}$. Эвтектическая точка существует при температуре $800 \mathrm{~K}$ и составе $15 \mathrm{~mol} \%$ германия. С учетом характера фазовых равновесий в двойных системах предложены две возможные схемы фазового субсолидусного разделения диаграммы состояний тройной системы. Выбор сделан на основании рентгенофазового анализа образцов политермического сечения $\mathrm{Sn}_{4} \mathrm{P}_{3}-\mathrm{GeP}$. Установлено, что сплавы разреза представляют смесь трех фаз - $\mathrm{Sn}_{4} \mathrm{P}_{3}$, GeP и Ge. Соответственно этому, разделение диаграммы ниже температуры солидуса осуществляют разрезы $\mathrm{Sn}_{4} \mathrm{P}_{3}-\mathrm{Ge}$, $\mathrm{Sn}_{4} \mathrm{P}_{3}-\mathrm{GeP}$ и $\mathrm{SnP}_{3}-\mathrm{GeP}$.

Таблица 1. Четырехфазные и трехфазные равновесия в тройной системе Ge - P - Sn

[Table 1. Four-phase and three-phase equilibria in the Ge - P - Sn ternary system]

\begin{tabular}{|c|c|c|c|}
\hline \multicolumn{2}{|c|}{$\begin{array}{c}\text { Нонвариантные равновесия } \\
\text { [Invariant equilibria] }\end{array}$} & \multicolumn{2}{|c|}{$\begin{array}{c}\text { Моновариантные равновесия } \\
\text { [Monovariant equilibria] }\end{array}$} \\
\hline $\begin{array}{l}\text { Точка } \\
\text { [the point] }\end{array}$ & $\begin{array}{c}\text { Процесс } \\
\text { [the process] }\end{array}$ & $\begin{array}{l}\text { Кривая } \\
\text { [the curve] }\end{array}$ & $\begin{array}{c}\text { Процесс } \\
\text { [the process] }\end{array}$ \\
\hline т. $\mathrm{E}_{1}$ & $\mathrm{~L} \leftrightarrow \mathrm{Sn}+\mathrm{Ge}+\mathrm{Sn}_{4} \mathrm{P}_{3}$ & $\mathrm{e}_{1} \mathrm{E}_{1}$ & $\mathrm{~L} \leftrightarrow \mathrm{Sn}_{4} \mathrm{P}_{3}+\mathrm{Sn}$ \\
\hline T. $\mathrm{E}_{2}$ & $\mathrm{~L} \leftrightarrow \mathrm{Sn}_{4} \mathrm{P}_{3}+\mathrm{SnP}_{3}+\mathrm{GeP}$ & $\mathrm{e}_{5} \mathrm{E}_{1}$ & $\mathrm{~L} \leftrightarrow \mathrm{Ge}+\mathrm{Sn}$ \\
\hline T. $E_{3}$ & $\mathrm{~L} \leftrightarrow \mathrm{SnP}_{3}+\mathrm{P}+\mathrm{GeP}$ & $e_{6} E_{1}$ & $\mathrm{~L} \leftrightarrow \mathrm{Ge}+\mathrm{Sn}_{4} \mathrm{P}_{3}$ \\
\hline \multirow[t]{6}{*}{ т. $\mathrm{U}_{1}$} & $\mathrm{~L}+\mathrm{Ge} \leftrightarrow \mathrm{Sn}_{4} \mathrm{P}_{3}+\mathrm{GeP}$ & $\mathrm{e}_{2} \mathrm{E}_{2}$ & $\mathrm{~L} \leftrightarrow \mathrm{Sn}_{4} \mathrm{P}_{3}+\mathrm{SnP}_{3}$ \\
\hline & & $\mathrm{e}_{3} \mathrm{E}_{3}$ & $\mathrm{~L} \leftrightarrow \mathrm{SnP}_{3}+\mathrm{P}$ \\
\hline & & $\mathrm{e}_{4} \mathrm{E}_{3}$ & $\mathrm{~L} \leftrightarrow \mathrm{GeP}+\mathrm{P}$ \\
\hline & & $\mathrm{e}_{6} \mathrm{U}_{1}$ & $\mathrm{~L} \leftrightarrow \mathrm{Sn}_{4} \mathrm{P}_{3}+\mathrm{Ge}$ \\
\hline & & $\mathrm{U}_{1} \mathrm{E}_{2}$ & $\mathrm{~L} \leftrightarrow \mathrm{Sn}_{4} \mathrm{P}_{3}+\mathrm{GeP}$ \\
\hline & & $\mathrm{pU}_{1}$ & $\mathrm{~L}+\mathrm{Ge} \leftrightarrow \mathrm{GeP}$ \\
\hline
\end{tabular}



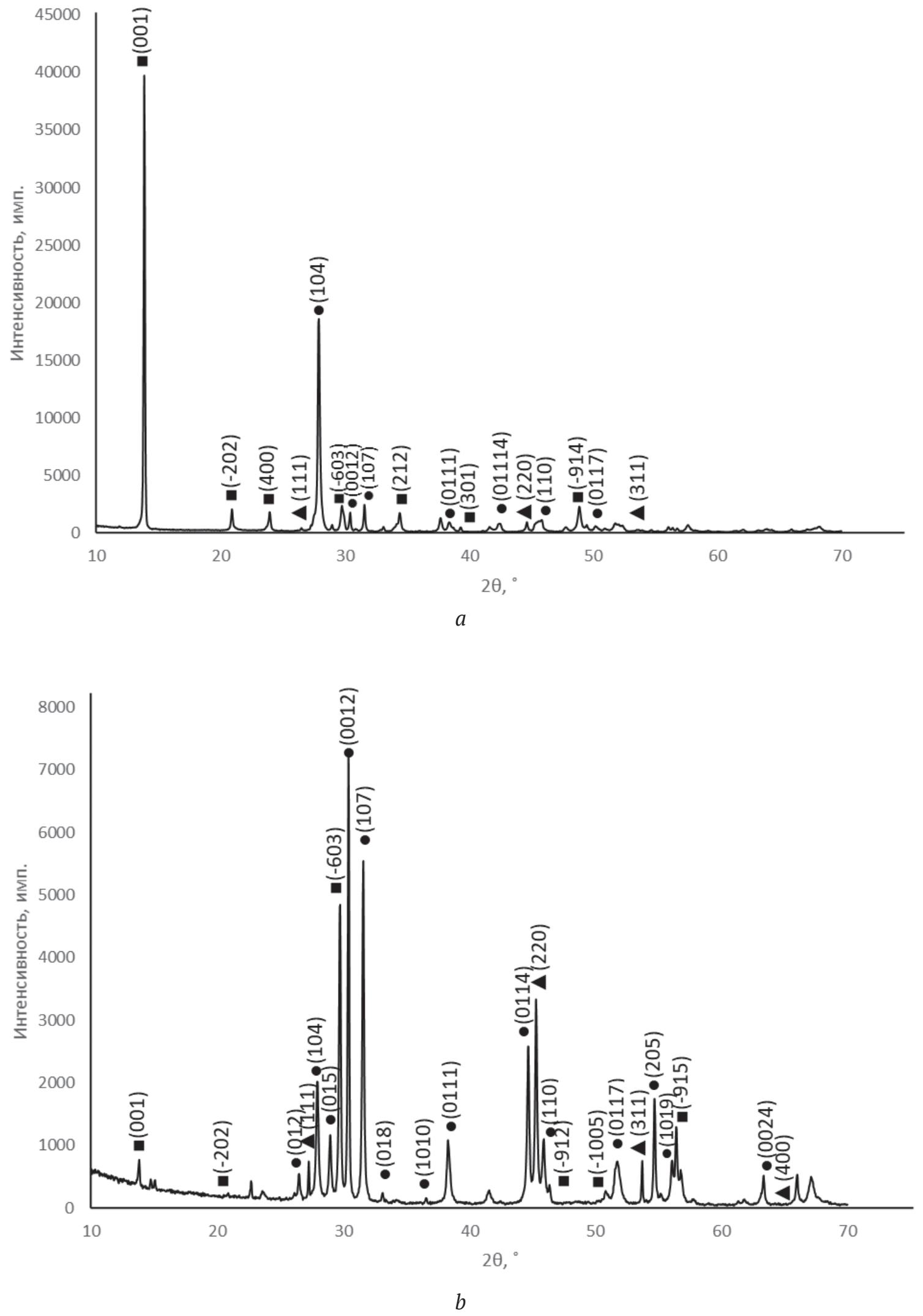

Рис. 5. Дифрактограммы сплавов политермического сечения $\mathrm{Sn}_{4} \mathrm{P}_{3}-\mathrm{GeP}: a-\left(\mathrm{Sn}_{4} \mathrm{P}_{3}\right)_{0.8} \mathrm{GeP}_{0.2}$; $b-\left(\mathrm{Sn}_{4} \mathrm{P}_{3}\right)_{0.6} \mathrm{GeP}_{0.3} ; c-\left(\mathrm{Sn}_{4} \mathrm{P}_{3}\right)_{0.4} \mathrm{GeP}_{0.5}$

[Fig. 5. X-ray powder diffraction patterns of alloys: $a-\left(\mathrm{Sn}_{4} \mathrm{P}_{3}\right)_{0.8} \mathrm{GeP}_{0.2} ; b-\left(\mathrm{Sn}_{4} \mathrm{P}_{3}\right)_{0.6} \mathrm{GeP}_{0.3}$; $\left.c-\left(\mathrm{Sn}_{4} \mathrm{P}_{3}\right)_{0.4} \mathrm{GeP}_{0.5}\right]$ 


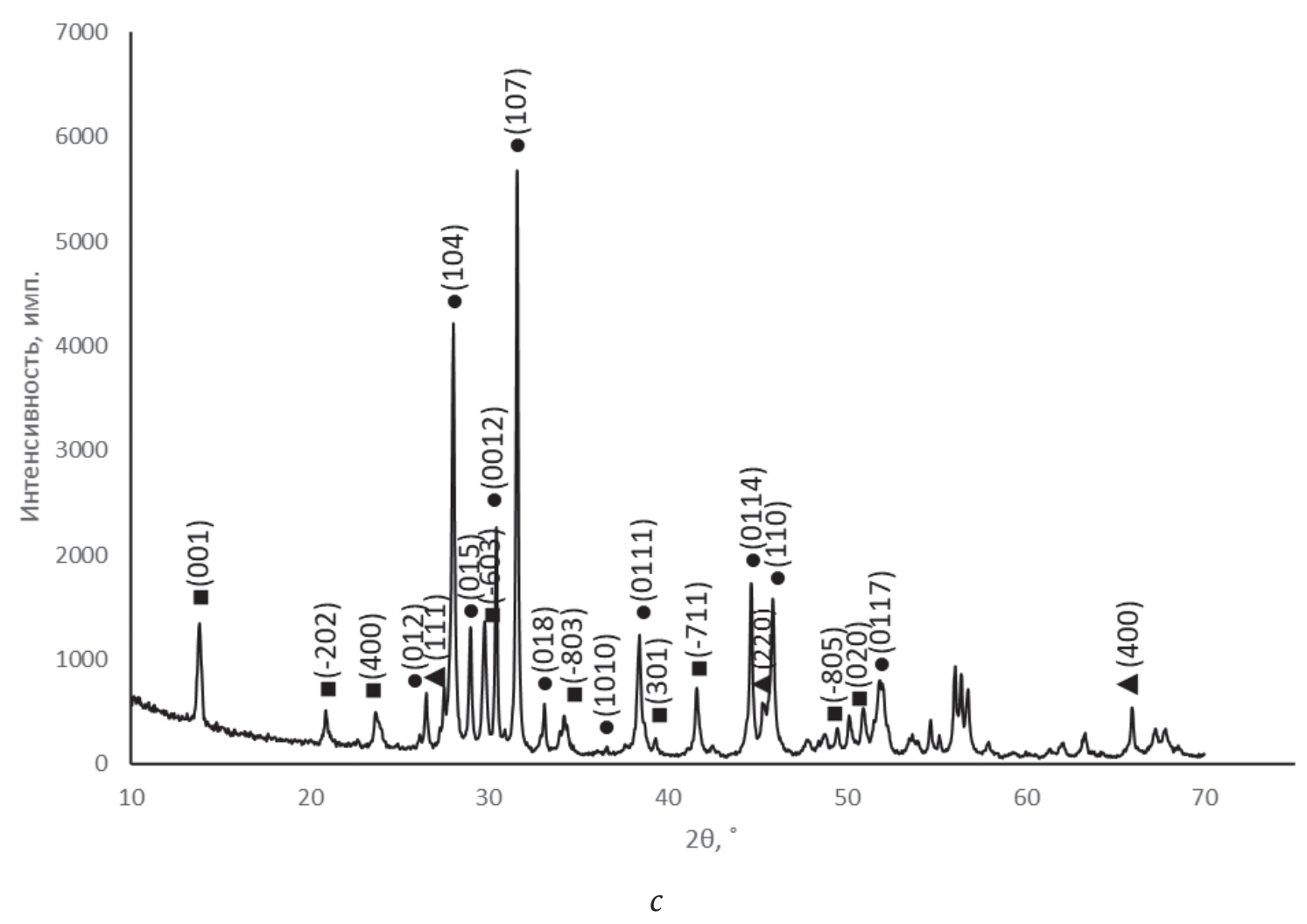

Рис. 5. Окончание

[Fig. 5. The end of the picture]

\section{БЛАГОДАРНОСТИ}

Результаты исследований получены на оборудовании Центра коллективного пользования научным оборудованием Воронежского государственного университета. URL: http://ckp. vsu.ru.

\section{КОНФЛИКТ ИНТЕРЕСОВ}

Авторы декларируют отсутствие явных и потенциальных конфликтов интересов, связанных с публикацией настоящей статьи.

\section{СПИСОК ЛИТЕРАТУРЫ}

1. Castellanos-Gomez A. Why all the fuss about 2D semiconductors? // Nature Photonics, 2016, v. 10, pp. 202-204. DOI: 10.1038/nphoton.2016.53

2. Hasan M. Z., Kane C. L. Colloquium: Topological insulators // Rev. Mod. Phys. 2010, v. 82, pp. 3045-3067. DOI: 10.1103/revmodphys.82.3045

3. Piot P., Behrens C., Gerth C., Dohlus M., Lemery F., Mihalcea D., Stoltz P., Vogt M. Erratum: Generation and Characterization of Electron Bunches with Ramped Current Profiles in a Dual-Frequency Superconducting Linear Accelerator // Phys. Rev. Lett., 2012, v. 108, pp. 1-5. DOI: 10.1103/physrevlett.108.229902
4. Dávila M.E., Xian L, Cahangirov S., Rubio A., Le Lay G. Germanene: a novel two-dimensional germanium allotrope akin to graphene and silicene // New J. Phys., 2014, v. 16, pp. 095002. DOI: $10.1088 / 1367-$ 2630/16/9/095002

5. Lalmi B., Oughaddou H., Enriquez H., Kara A., Vizzini S., Ealet B., Aufray B. Epitaxial growth of a silicene sheet // Appl. Phys. Lett., 2010, v. 97, pp. 223109. DOI: $10.1063 / 1.3524215$

6. Kara H., Enriquez H., Seitsonen Ari P., Lew Yan Voon L.C., Vizzini S., Aufray B., Oughaddou H. Corrigendum to "A review on silicene - New candidate for electronics” // Surf. Sci. Rep., 2012, v. 67, pp. 1-18.DOI: 10.1016/j.surfrep.2012.01.001

7. Barreteau C., Michon B., Besnard C., Giannini E. High-pressure melt growth and transport properties of SiP, SiAs, GeP, and GeAs 2D layered semiconductors // J. Cryst Growth., 2016, v. 443, pp. 75-80. DOI: 10.1016/j.jcrysgro.2016.03.019

8. Угай Я. А., Соколов Л. И., Гончаров Е. Г. Р-Т-Х диаграмма состояния системы $\mathrm{GeP}$ и термодинамика взаимодействия компонентов // Ж. неорганической химии, 1978, т. 23(7), с. 1907-1911.

9. Lee K., Synnestvedt S., Bellard M., Kovnir K. GeP and $(\mathrm{Ge} 1-\mathrm{Sn})(\mathrm{P} 1-\mathrm{Ge})(\mathrm{x} \approx 0.12, \mathrm{y} \approx 0.05)$ : Synthesis, structure, and properties of two-dimensional layered tetrel phosphides // J. Solid State Chem., 2015, v. 224, 
pp. 62-70. DOI: https://doi.org/10.1016/j. jssc.2014.04.021 336.

10. Vivian A. C. // J. Inst. Met, 1920, v. 23, pp. 325-

11. Zavrazhnov A. Yu., Semenova G. V., Proskurina E. Yu., Sushkova T. P. Phase diagram of the Sn-P system // J. Thermal Analysis and Calorimetry, 2018, vol. 134(1), pp. 475-481. DOI: 10.1007/s10973-0187123-0

12. Olesinski R. W., Abbaschian G. J. The Ge-Sn (Germanium-Tin) system // Bulletin of Alloy Phase
Diagrams, 1984, v. 5(3), pp. 265-271. DOI: $10.1007 /$ bf02868550

13. Глазов В. М., Павлова Л. М. Химическая термодинамика и фазовые равновесия. М.: Металлургия, 1988,560 c.

14. Эмсли Дж. Элементы. М.: Мир, 1993. с. 5657.

15. Arita M. Kamo K. Measurement of Vapor Pressure of Phosphorus over Sn-P Alloys by Dew Point Method // Trans. Jpn. Inst. Met, 1985, v. 26(4), pp. 242250. DOI: $10.2320 /$ matertrans 1960.26 .242

UDC 541.123.3

DOI: https://doi.org/10.17308/kcmf.2019.21/763

Received 19.04.2019

Accepted 15.05.2019

\title{
ANALYSIS OF PHASE EQUILIBRIA IN THE Ge-P-Sn TERNARY SYSTEM
}

\author{
(c) 2019 G. V. Semenova $₫$, T. A. Leontyeva, T. P. Sushkova \\ Voronezh State University \\ 1, Universitetskaya pl., 394018 Voronezh, Russian Federation
}

\begin{abstract}
Objective. Great attention to compounds with a layered structure because of the possibility of formation of functional 2D materials on their basis predetermined the interest in compounds of the group $\mathrm{A}^{\mathrm{IV}} \mathrm{B}^{\mathrm{V}}$, in particular GeP. However, the high vapor pressure of phosphorus creates the complexity of the synthesis of this phase, so they use a solvent - tin or bismuth, which significantly softens the conditions for obtaining samples. In this case, other phases may form, according to the nature of the equilibria in the ternary systems. This determines the relevance of research on the nature of phase equilibria in $\mathrm{Ge}-\mathrm{P}-\mathrm{Sn}$ ternary system. The purpose of this paper is to analyze the phase diagram of the $\mathrm{Ge}-\mathrm{P}-\mathrm{Sn}$ system and possible equilibrium schemes that take into account the features of binary systems that bounding this ternary system, based on an experimental study of the $\mathrm{Sn}_{4} \mathrm{P}_{3}-\mathrm{Ge}$ and $\mathrm{Sn}_{4} \mathrm{P}_{3}-\mathrm{GeP}$ polythermal cross sections.

Methods and Methodology. The samples were obtained by fusing simple substances of tin, germanium and red phosphorus in evacuated quartz ampoules. Then the alloys were annealed at a temperature $753 \mathrm{~K}$ for 120 hours. The study of the samples was carried out on the differential thermal analysis (DTA) setup with a programmable heating of the furnace. In our experiments, the heating rate of DTA-setup was $5 \mathrm{~K} \cdot \mathrm{min}^{-1}$. X-ray powder diffraction (XRD) analysis of samples was performed using a powder diffractometer ARL X'TRA with the following characteristics: $\mathrm{CuK} \alpha$-radiation, exposure step $0.06^{\circ}$, exposure time 3.0 seconds.

Results. As follows from the data of differential thermal analysis, the phase diagram of the $\mathrm{Sn}_{4} \mathrm{P}_{3}-\mathrm{GeP}$ section is a diagram of the eutectic type with the coordinates of the eutectic point of $800 \mathrm{~K}, 15 \mathrm{~mol} \% \mathrm{Ge}$. An experimental study by the X-ray phase analysis of alloys whose compositions correspond to polythermal section $\mathrm{Sn}_{4} \mathrm{P}_{3}-\mathrm{GeP}$ made it possible to establish the nature of the phase separation of the system at temperatures below the solidus. In the system invariant peritectic equilibrium $\mathrm{L}+\mathrm{Ge} \leftrightarrow \mathrm{GeP}+\mathrm{Sn}_{4} \mathrm{P}_{3}$ takes place, and there are also three triple eutectic points, responding to the processes $\mathrm{L} \leftrightarrow \mathrm{Ge}+\mathrm{Sn}+\mathrm{Sn}_{4} \mathrm{P}_{3} ; \mathrm{L} \leftrightarrow \mathrm{GeP}+\mathrm{SnP}_{3}+\mathrm{Sn}_{4} \mathrm{P}_{3}$ and $\mathrm{L} \leftrightarrow \mathrm{P}+\mathrm{GeP}+\mathrm{SnP}_{3}$

Conclusions. Taking into account the nature of phase equilibria in binary systems, two possible schemes of sub-solidus phase separation of the $\mathrm{Ge}-\mathrm{P}-\mathrm{Sn}$ ternary system diagram are proposed. The choice was made on the basis of X-ray phase analysis of samples of polythermal section
\end{abstract}

$\checkmark$ Semenova Galina V., e-mail: semen157@chem.vsu.ru 
$\mathrm{Sn}_{4} \mathrm{P}_{3}-\mathrm{GeP}$. It was established that the sectionrs alloys are a mixture of three phases $-\mathrm{Sn}_{4} \mathrm{P}_{3}, \mathrm{GeP}$ and Ge. Accordingly, the separation of the diagram below the solidus temperature is performed by $\mathrm{Sn}_{4} \mathrm{P}_{3}-\mathrm{Ge}, \mathrm{Sn}_{4} \mathrm{P}_{3}-\mathrm{GeP}$ and $\mathrm{SnP}_{3}-\mathrm{GeP}$ cuts.

Keywords: phase equilibria, Ge - P - Sn ternary system, of sub-solidus phase separation.

\section{ACKNOWLEDGMENTS}

The research results were obtained using the equipment of the Center for Collective Use of Equipment Voronezh State University. URL: http:// ckp.vsu.ru.

\section{CONFLICT OF INTEREST}

The authors declare the absence of obvious and potential conflicts of interest related to the publication of this article.

\section{REFERENCES}

1. Castellanos-Gomez A. Why all the fuss about 2D semiconductors? Nature Photonics, 2016, v. 10, pp. 202-204. DOI: 10.1038/nphoton.2016.53

2. Hasan M. Z., Kane C. L. Colloquium: Topological insulators. Rev. Mod. Phys., 2010, v. 82, pp. 3045-3067. DOI: 10.1103/revmodphys.82.3045

3. Piot P., Behrens C., Gerth C., Dohlus M., Lemery F., Mihalcea D., Stoltz P., Vogt M. Erratum: Generation and Characterization of Electron Bunches with Ramped Current Profiles in a Dual-Frequency Superconducting Linear Accelerator. Phys. Rev. Lett., 2012, v. 108, pp. 1-5. DOI: 10.1103/physrevlett.108.229902

4. Dávila M. E., Xian L, Cahangirov S., Rubio A., Le Lay G. Germanene: a novel two-dimensional germanium allotrope akin to graphene and silicene. New J. Phys., 2014, v. 16, pp. 095002. DOI: 10.1088/13672630/16/9/095002

5. Lalmi B., Oughaddou H., Enriquez H., Kara A., Vizzini S., Ealet B., Aufray B. Epitaxial growth of a silicene sheet. Appl. Phys. Lett., 2010, v. 97, pp. 223109. DOI: 10.1063/1.3524215

6. Kara H., Enriquez H., Seitsonen Ari P., Lew Yan Voon L.C., Vizzini S., Aufray B., Oughaddou H. Corrigendum to "A review on silicene - New candidate for electronics”. Surf. Sci. Rep., 2012, v. 67, pp. 1-18. DOI: 10.1016/j.surfrep.2012.01.001
7. Barreteau C, Michon B, Besnard C, Giannini E. High-pressure melt growth and transport properties of SiP, SiAs, GeP, and GeAs 2D layered semiconductors. J. Cryst Growth., 2016, v. 443, pp. 75-80. DOI: 10.1016/ j.jcrysgro.2016.03.019

8. Ugai Ya. A., Sokolov L.I., Goncharov E.G. P-T-X diagramma sostoyaniya sistemy GeP i termodinamika vzaimodeystviya komponentov [P-T-X GeP system state diagram and thermodynamics of component interaction] // Russian Journal of Inorganic Chemistry, 1978, v. 23(7), pp. 1907-1911. (in Russ.)

9. Lee K., Synnestvedt S., Bellard M., Kovnir K. GeP and $(\mathrm{Ge} 1-\mathrm{Sn})(\mathrm{P} 1-\mathrm{Ge})(\mathrm{x} \approx 0.12, \mathrm{y} \approx 0.05)$ : Synthesis, structure, and properties of two-dimensional layered tetrel phosphides. J. Solid State Chem., 2015, v. 224, pp. 62-70. DOI: https://doi.org/10.1016/j. jssc.2014.04.021 336.

10. Vivian A. C. J. Inst. Met, 1920, v. 23, pp. 325-

11.Zavrazhnov A. Yu., Semenova G. V., Proskurina E. Yu., Sushkova T.P. Phase diagram of the Sn-P system. J. Thermal Analysis and Calorimetry, 2018, v. 134(1), pp. 475-481. DOI: 10.1007/s10973-018-7123-0

12. Olesinski R. W., Abbaschian G. J. The Ge-Sn (Germanium-Tin) system. Bulletin of Alloy Phase Diagrams, 1984, v. 5(3), pp. 265-271. DOI: 10.1007/ bf02868550

13. Glazov V. M., Pavlova L. M. Khimicheskaya termodinamika i fazovyye ravnovesiya [Chemical thermodynamics and phase equilibria]. Moscow, Metallurgiya Publ, 1988, 560 p. (in Russ.)

14. Emsley J. The elements: Second Edition. Oxford University Press, Oxford, 1991.

15. Arita M. Kamo K. Measurement of Vapor Pressure of Phosphorus over Sn-P Alloys by Dew Point Method. Trans. Jpn. Inst. Met, 1985, v. 26(4), pp. 242250. DOI: $10.2320 /$ matertrans1960.26.242
Семенова Галина Владимировна - д. х. н., профессор кафедры общей и неорганической химии, Воронежский государственный университет, Воронеж, Российская Федерация; e-mail: semen157@chem.vsu.ru. ORCID iD 0000-00033877-985X.
Semenova Galina V. - Dr. Sci. (Chem.), Full Professor, Department of General and Inorganic Chemistry, Voronezh State University, Voronezh, Russian Federation; e-mail: semen157@ chem.vsu.ru.ORCID iD 0000- 0003-3877-985X. 
Леонтьева Татьяна Андреевна - студентка 5 курса химического факультета. Воронежский государственный университет, Воронеж, Российская Федерация; e-mail: tanya_leonteva090297@ mail.ru. ORCID iD 0000-0001-9860-2234.

Сушкова Татьяна Павловна - к. х. н., доцент кафедры общей и неорганической химии, Воронежский государственный университет, Воронеж, Российская Федерация; e-mail: sushtp@ yandex.ru. ORCID iD 0000-0003-1969-7082
Leont'eva Tat'yana A. - 5th year student of the Faculty of Chemistry, Voronezh State University, Voronezh State University, Voronezh, Russian Federation; e-mail: tanya_leonteva090297@mail.ru. ORCID iD 0000-0001-9860-2234.

Sushkova Tat'yana P. - Cand. Sci. (Chem.), Assistant Professor, Department of General and Inorganic Chemistry, Voronezh State University, Voronezh, Russian Federation; e-mail: sushtp@yandex.ru. ORCID iD 0000-0003-1969-7082. 